\title{
Transient receptors potential (TRP) channels and neuroimmunology Mutation pathways of Rosacea.
}

\author{
Chan KTM* \\ Specialist in Dermatology, Hong Kong Academy of Medicine, Hong Kong.
}

\begin{abstract}
Rosacea is a complicated skin disease with a plethora of phenotypic manifestations. Environmental factors, skin barrier dysfunction, epidermal antimicrobial peptides, demodicosis, neurovascular dysfunction are all believed to play a role. TRP channels and its associated proteins interactors and immunological pathways may play a significant role $n$ the pathogenesis of Rosacea.
\end{abstract}

Keywords: Transient receptors potential (TRP) channels, Pathogenesis, Rosacea.

Accepted on 08 May, 2018

\section{Introduction}

Rosacea is a complicated skin disease with diverse phenotypic manifestations [1-4]. Sensitive skin is believed to be associated with Rosacea [5,6]. Environmental factors, skin barrier dysfunction, epidermal antimicrobial peptides, demodicosis, neurovascular dysfunction are all believed to play a role. With the advent of neuro immunology, the pivotal roles of the TRP channels in the pathogenesis of Rosacea become apparent [7-10] TRPV1, TRPV2, TRPV3, TRPV4, TRPA1 with its associated protein interactors like $G$ Protein $C$ Receptor (GPCR) and inflammasone producing Toll like receptors and LL-37 are all involved.

TRP are proteins channels known to be widely distributed in most of the body tissue include the epidermis, peripheral and central nervous system, endothelium and smooth muscles of blood vessels, the dermal inflammatory cells and even gut $[6,8,9]$. TRP channels provide good molecular signalling pathways in linking up most of the immunological pathogenetic events leading to Rosacea. Increased cutaneous blood flow during excessive warmth, high temperature and heat initially responded by vasodilatation mediated by axonal reflexes and the secondary slow responding vasodilatation to nitric oxide (NO) [10]. In addition, Rosacea patients showed an altered control of vascular endothelial cells which increase the blood flow. Enhanced and unimpeded expression of TRPVs proteins result in flushing, vaso-dysregulation, neurogenic and nonspecific inflammation. Immunostaining with TRPV showed strong positivity in the dermal inflammatory cells in Rosacea. TRPV gene expressions were increased in Rosacea [11]. Each TRPV channels appeared to be activated by heat and acted through its own polymodal neuro-immunoendocrine mechanism [8-9].

\section{TRPV1}

TRPV 1 is distributed in keratinocytes, free nociceptive sensory nerve endings among epidermis and dermis, inflammatory cells like mast cells, Langerhans Cells, fibroblasts, endothelial cells, sebocytes in follicular appendages, dorsal root ganglion of the spinal cord and the central nervous system $[6,8,9]$. In Rosacea, it has been established aberrant sensation respond to heat, exercises, stress and spices are TRPV 1 mediated with clinically presentations of flushing, stinging, burning pain and itch. This resemble the sensitive skin pathogenesis which is believed to be TRPV mediated [5,6]. The role of TRPV1 in Rosacea has been supported by immunostaining of TRPV 1 protein in the epidermis and TRPV 1 mRNA expression reported in Rosacea. In sensitive skin; both dry skin and altered neurovascular responsiveness has been suggested to be a result of hyperreactivity of TRPV 1 [11]

Persistent activation of TRPV 1 translate into neurogenic inflammatory cascades with the release of pro-inflammatory cytokines like interleukin-1 (IL-1), interleukin-8 (IL-8), Prostaglandin E2 (PEG2), Transforming growth factors $\beta 2$ (TGFb2) and Matrix metalloproteinases 1 (MMP1) [6,8]. TRPV 1 activation leaded to the secretions of Substance P (Sub $\mathrm{P}$ ) in the dorsal root ganglion neurons, SP is known to be a potent noxious signal neurotransmitter which triggers the glutamate pathway and by calcium influx through the $\mathrm{N}$ methyl-D-aspartate (NMDA) receptor with the production of sensation of itch $[6,8]$. Apart from SP, the pruritogen like Calcitonin Gene Related Peptide (CGRP) and Tachykinin were also produced. SP will also provoke a viscous cycle of local tissue inflammatory responses through a number of mediators and cytokines [6,8]. TRPV1 regulate sebocytes activity especially in sebum secretion and inflammation [8]. Hence, TRPV 1 may well be involved in the early stages of development of all forms of Rosacea especially in the inflammatory stage and sensory aggregating phase.

\section{TRPV 2}

TRPV 2 expression has been reported in a wide range of immune and inflammatory cell types with the release of cytokines, chemo taxis of white cells, phagocytes and inflammasone activity [8]. TRPV 2 protein was shown positive in immunostaining in both macrophages and mast cells in Rosacea setting [11]. It is activated by high temperature, calcium influx and insulin Growth Factor Signalling [8]. Evidences suggested that continuous stimulation of TRPV 2 may promote neurite outgrowths. Allokinesis in response to mechanical stretch of neurons or nerve growth factor (NGF) stimulation are thought to be TRPV 2 mediated [12]. 


\section{TRPV 3}

TRPV 3 channels are located on the surface of keratinocytes was activated by heat with the release of the vasoactive nitric oxide (NO) from keratinocytes and this is independent of the action of NO synthetase enzymatic pathway [13]. TRPV 3 also stimulates keratinocytes to release Prostaglandin E2 (PGE 2) [14]. Both NO and PEG2 affect not only neuronal excitation but more importantly has an effect of vasodilation. This suggested that TRPV 3 may have a pathogenetic role in translating noxious signals to phenotypic manifestations of Rosacea. TRPV 3 over-reactivity will produce an epidermal protease mediated shedding of Epidermal Growth Factor Release (EGFR) ligand called (Transforming Growth Factors) TGF- $\alpha$. TRPV 3 also promotes transglutaminase activity in keratinocyte with the result of skin barrier defects seen in dry skin of Rosacea [15].

\section{TRPV 4}

TRPV 4 is closely linked to the pathogenesis of the different phenotypic presentations of Rosacea. Cathelicidin; a potent antibacterial protein; secreted by keratinocyte in response to antimicrobial invasion; is produced through the Kallikrein 5 pathway involving the Matrix metalloproteinases (MMP) resulted LL-37. LL-37 also known as defensins upregulate TRPV 4 in vivo and activate mast cells in a dose dependent manner [16]. Known inhibitors of TRPV 4 have been shown in study to reverse and stop this activation. LL-37 has been demonstrated to be a major pathogenetic factor in causing Rosacea through inflammation and angiogenesis of skin [17]. This important link in the pathogenesis of Rosacea is also TRP channels mediated through its cellular host; the mast cells. TRPV 4 integrates signalling pathway to a wide variety of stimuli. Inflammatory mediators like bradykinin, PGE2, Sub P, Serotonin, histamine, Proteases Activator Receptors 2 (PAR2) causing neuro-immunological reaction in the skin and other body organs like the brain and cardiovascular systems.

TRPV 4 is a key regulator of epithelial and endothelial barrier function of blood vessels. TRPV 4 located on the primary afferent nociceptive nerves is thought to function as an osmo transducer, thereby mediating the nociceptive response to hypotonic stimulation [18]. Sheer stress-induced vasodilation is critically dependent on endothelial TRPV 4 expression. Sheer stress opens TRPV 4 to increase intracellular calcium and vascular endothelial cells [18]. TRPV 4 activation is essential for activation of $\mathrm{B} 1$ integrins in response to mechanical stress in capillary endothelial cells. The vascular endothelium secretes factors that control vessel tone in response to vasoactive factors and sheer stress elicited by blood flow. TRPV 4 is expressed both in the endothelium and smooth muscle cells of the vasculature [18]. It is shown that activation of TRPV 4 channels has been shown to mediate local calcium influx and sheer stress induced vasodilatation and cause maximal dilatation [18]. Recently, GPCR; a TRP channel protein interactor; was shown to open TRPV 4 through a signalling pathway involve serotonin, PAR 4 and mechanoreceptors which may include arachidonic acid metabolites like, EET and protein kinases [19].
Epoxyeicosatrienocic acid (EET) metabolite of arachidonic acid; polyunsaturated omega- 6 fatty acid; causes vascular smooth muscle vasodilatation and relaxation through depolarization. This is NO and prostacyclin independent [20]. Strong evidence suggested that TRPV 4 opening by a muscarinic receptor dependent mechanism in various vascular beds. Receptor dependent opening of TRPV 4 has been observed in acetylcholine induced vasodilatation in cerebral, mesenteric and skeletal muscle [21].

TRPV 4 is important in relaying and translating internal signals, inflammatory mediators, neurogenic information to results different patho-immunological disease state like vasodilatation, inflammation, pain, itch and other systemic effects involving the brain, gut and cardiovascular system. TRPV 4 has a crucial role on the phenotypic manifestations of Rosacea. Last not the least, TRPV 4 has also a significant role in the pathogenesis of fibrosis which was commonly seen in end-stage Rosacea patients in Rhinophyma. TRPV 4 is a major regulator in the progression of fibrosis. Fibrosis is characterized by excessive deposition of connective tissues in conjunction with a reactive process. Inflammation is a prodrome of fibrosis. Accumulation of myofibroblasts with deposition of extracellular matrix connective tissues which is metallo matrix proteinases (MMPs) regulated and fibroblast activation include Transforming growth factors B (TFG B), cytokines, TNF-, PDGF and interleukin and signalling pathways like SMAD, Rho/ROCK, ERK and P13K/AK) may all been involved. Fibrosis can be seen in heart, kidney, lung, liver and skin are closely linked with TRPV 4 regulation.

\section{TRPA 1}

TRPA 1 is abundantly distributed in nociceptive sensory neurones and numerous inflammatory cell types particularly mast cells $[6,8]$. TRPA 1 is also a heat and mechanical neuro receptors. Interestingly, unlike TRPV1, TRPA 1 was reported to have $U$ shaped temperature response profile secondary to activation of both cold and heat. This is believed to be due to TRPA1 regulation by redox state modulation. 8 TRPA1 is upregulated by the pathogen activator Toll Like Receptor 7 (TLR 7) which is an inflammasone activated complex of inflammation.

Pathologically, TRPA1 is actively involved in sensory dysfunction especially in acute and chronic itch. Studies suggested that the pruritogen sensing $G$ protein-coupled receptors: MrgprA3 and MrgC11 ligands associated nonhistaminergic itch is TRPA1 mediated. HTR 7 and TRPA1 are functionally coupled in eliciting itch. Hydroxytryptamine 7 (HTR 7) receptor activation triggers Serotonin then in turn promote opening of the TRPA1 channels in eliciting itch in the CNS [22]. IL-31 was also closely assembled both anatomically and functionally with TRPA1. IL-31 receptors were found to be colocalised with TRPA1 and TRPV 1 in sensory neurons and genetic elimination of either reduced IL-31 itch [23]. TSLP act on epidermal keratinocytes in response to histaminergic signalling activates neuronal TRPA 1 downstream of TSLP receptors. In inflammation and epidermal homeostasis; TRPA 1 depicted mice model bench side in vivo studies; a suppressed 
Citation: Chan Michael. Transient receptors potential (TRP) channels and neuroimmunology Mutation pathways of Rosacea. J Cell Sci Mut 2018;2(1):5.

cytokine response and reduction in hallmarks inflammatory markers with a reduction of epidermal thickening was observed [24]. In vascular tone activity, TRPA1 possessed neurons located in the spinal cord of the dorsal horn of CNS when stimulated was shown to release pro-inflammatory peptides like SP and CGRP into their target tissues with subsequent vasodilatation and extravasation [25]. TRPA 1 is also pathogenetically involved in Rosacea resulting sensory dysfunction, pronounced inflammation, vascular instability and systemic consequences especially in the CNS with Serotonin involvements.

\section{Conclusion}

In sum, TRP channel proteins are actively involved in the pathogenesis and immunological events of Rosacea. TRPV 1, TRPV2 TRPV 3 and TRPA 1 are receptors of the sensation of heat while TRPA 1 and TRPM 8 are neuro receptors of cold. Most of the TRP members described: TRPV1, TRPV2, TRPV 3 , TRPV 4, TRPA1 are involved in the inflammatory cascades neurogenically or/and non-neurogenically; locally or/and centrally in Rosacea. While TRPV 3 mediates vasodilatation via NO; TRPV 4 phosphorylation has more systemic independent vascular tone modulatory effects affecting the vessels. TRPA 1 activity on vascular tone mediates through inflammatory neuronal signals in the spinal cord. Moreover, the involvement of TRPV 4 in body innate anti-microbial defence through Cathelicidin and organ fibrosis provide further evidence of the importance of TRP channels in Rosacea.

\section{References}

1. Schaller M, Almeida LM, Bewley A, et al. Rosacea treatment update: recommendations from the global ROSacea Consensus (ROSCO) panel. $\mathrm{Br} \mathrm{J}$ Dermatol. 2017;176(2):465-71.

2. Rainer BM, Kang S, Chien AL, et al. Rosacea: Epidemiology, pathogenesis and treatment. Dermatoendocrinology. 2018;9(1):1361-574.

3. Chan KTM. Severe Acneiform Facial Eruption: An Updated Prevention, Pathogenesis and Management. Med Clin Res. 2017;2(2):1-5.

4. Rainer BM, Fischer AH, Luz Felipe da Silva D, et al. Rosacea is associated with chronic systemic diseases in a skin severity-dependent manner: results of a case-control study. JAAD. 2015;73:604-08.

5. Misery L, Loser K, Stander S, et al. Sensitive skin. J Eur Acad Dermatol Venereol 2016;1:2-8.

6. Chan KTM. The Pivotal Role of Transient Receptor Potential (TRP) Ion Channels in the Pathogenesis of Sensitive Skin. Research J of Nervous System. 2018;1:1.

7. Two AM, Wu W, Gallo RL, et al. Rosacea: part I. Introduction, categorization, histology, pathogenesis, and risk factors. Pathways known to contribute to the pathophysiology and clinical manifestations of Rosacea. JAAD. 2018;72:749-58.

8. Caterina MJ, Pang Z TRP Channels in Skin Biology and Pathophysiology. Pharmaceuticals. 2016;9.
9. Toth BI, Olah A, Szollosi AG, et al. TRP channels in the skin. British Journal of Pharmacology. 2014;171:2568-81.

10. Yu RW Rosacea, molecular mechanism and management of a chronic cutaneous inflammatory condition. Int $\mathrm{J}$ of Molecular Science. 2016;17(9):15-62.

11. Sulk M, Seeliger S, Aubert J, et al. Distribution and expression of non-neuronal transient receptor potential (TRPV) ion channels in rosacea. J. investing. Dermatol. 2012;132:1253-62.

12. Cohen MR, Johnson WM, Pilat JM, et al. Nerve growth factor regulates Transient receptor potential Vanilloid 2 via extracellular signal-regulated kinase signalling to enhance neurite outgrowth in developing neurones. Mol Cell Biol. 2015;35(24): 4238-52.

13. Miyamoto T, Petrus MJ, Dubin AE, et al. TRPV3 regulates nitric oxide synthase-independent nitric oxide synthesis in the skin. Nat. Commun. 2011;2:369.

14. Huang SM, Lee H, Chung MK, et al. Overexpresssed transient receptor potential vanilloid 3 ion channels in skin keratinocytes modulate pain sensitivity via prostaglandin E2. J Neuroscience PEG2. J Neuroscience. 2018;28:13727-37.

15. Cheng X, Jin J, Hu L, et al. TRP channels regulates EGFR signalling in hair morphogenesis and skin barrier formation. Cell. 2010;141:331-43.

16. Mascarenhas NL, Wang Z, Chang Y, et al. TRPV 4 mediates mast cell activation in Cathelicidin-induced rosacea inflammation. J Invest Dermatology. 2017;137(4): 972-75.

17. Chen Y, Moore CD, Zhang JY, et al. TRPV 4 moves toward center-fold in rosacea pathogenesis. J Invest Dermatol. 2017;137(4): 801-04.

18. Darby WG. Modulation of TRPV 4 by diverse mechanism. The International Journal of Biochemistry \& Cell Biology. Sept, 2016;78:217-28.

19. Saifeddine M, Elpdaly M, Mihara k, et al. GPCR-mediated EGF receptor transactivation regulates TRPV4 action in the vasculature. Br J Pharmacol. May; 2015;172(10):2493-506.

20. Campbell WB, Fleming I. Epoxyeicosatrienoic acids and endothelium-dependent responses. Pflugers Arch. 2010;459:881-59.

21. Adapale RK, Talasila PK, Bratz IN, et al. PKC alpha mediates acetylcholine induced activation of TRPV 4 dependent influx in endothelial cells. Am J. Physiol. Heart Circ. Physiol. 2011;301:757-65.

22. Wilson SR, Gerhold KA, Bifolck-Fisher A, et al. TRPA1 is required for histamine -independent Mas-related $G$ proteincoupled receptor mediated itch. Nat Neurosci. 2011;14:595-602.

23. Wilson SR, The L, Batia LM, et al. The epithelial cellderived atopic dermatitis cytokine TSLP activates neurons to itch. Cell. 2013;155:285-95.

24. Lieu B, Escalera J, Balakrishna S, et al. FASEB J. TRPA 1 controls inflammation and pruritogen responses in allergic contact dermatitis. 2013;27:3549-63. 
25. Geppetti P, Nassini R, Materazzi s, et al. The concept of neurogenic inflammation. BJU International. 2008;101(3):

$2-6$.

\section{*Correspondence to:}

Chan KTM

Specialist in Dermatology

Hong Kong Academy of Medicine

Hong Kong

Tel: +852-21282129

E-mail: pioneerskin@ymail.com 